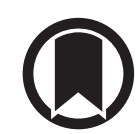

CrossMark

\section{A re-analysis in exploring the association between temperature and COVID-19 transmissibility: an ecological study with 154 Chinese cities}

To the Editor:

Exploring the role of ambient temperature in coronavirus disease 2019 (COVID-19) transmission is of importance in understanding the patterns of the epidemic. YAO et al. [1] concluded that there were no significant associations of COVID-19 transmissibility with temperature and ultraviolet (UV) radiation in 62 Chinese cities. Inspired by the nonlinear dose-response relationship between ambient temperature and influenza transmissibility explored previously $[2,3]$, we argue that the association between COVID-19 transmissibility and temperature might be complex in nature, which may be overlooked by a linear analytical framework. We, therefore, examined the likelihood of a nonlinear association between COVID-19 transmissibility and ambient temperature.

To quantify the COVID-19 transmissibility, we calculated $R_{0}$ for each Chinese city via the standard approach with a Gamma distribution having mean \pm SD values of $5.5 \pm 3.3$ days for the generation interval $[4,5]$. A total of 154 Chinese cities were detected with COVID-19 outbreaks $\left(R_{0}>1\right)$ between December 2019 and February 2020, the same period studied by YAO et al. [1]. Our $R_{0}$ estimates for the 154 cities (15 inside Hubei and 139 outside Hubei) have a mean \pm SD of $1.4 \pm 0.3$, which was largely consistent with the work reported by YaO et al. [1], where 62 Chinese cities were included (12 inside Hubei and 50 outside Hubei) [1]. The maximal $R_{0}$ was estimated at 2.5 (95\% CI 2.4-2.6) in Wuhan, which is consistent with previous findings $[1,5]$. We interpolated monitoring data of mean temperature and relative humidity during the same period from 689 stations to continuous rasters by ArcGIS, and then we extracted values from the rasters and linked them to the 154 selected cities.

We first used the multivariable linear regression model, the same framework with YAO et al. [1], and adjusted for relative humidity, to estimate the association between ambient temperature and $R_{0}$. We found the association was slightly negative but not of statistical significance (figure 1a), which is partially in line with YAO et al. [1] and another recent study across 100 Chinese cities [6]. Next, generalised linear regression with structural break design, i.e. discontinuity regression, was employed to explore the linear structural change in the relationship, in terms of the slope changes, between ambient temperature and $R_{0}$. The discontinuous point was selected based on the likelihood profile, shown in the inserted panel in figure 1b. We estimated the optimal discontinuous point at $7^{\circ} \mathrm{C}\left(95 \% \mathrm{CI}-7.0\right.$ to $\left.11.5^{\circ} \mathrm{C}\right)$, which is largely consistent with an emerging report across 429 cities worldwide [7]. In figure $1 \mathrm{~b}$, the temperature is negatively and significantly associated with COVID-19 transmissibility when it is higher than $7^{\circ} \mathrm{C}$. We further remarked that a slight change in the temperature discontinuous point would not affect the results. Then, for validation of the "shape of the relationship", we attempted multivariable nonlinear regression by using the natural spline function with two degrees of freedom determined by the minimal Akaike information criterion (AIC). We set the knot of the spline regression as the discontinuous point at $7^{\circ} \mathrm{C}$. The discontinuity regression model $(\mathrm{AIC}=51.78)$ and spline regression model $(\mathrm{AIC}=53.41)$ outperformed the linear regression model with smaller AICs (against the AIC of 53.82 for the linear regression). The overall nonlinear association between $R_{0}$ and temperature was of statistical significance in terms of the p-value $(=0.044)$ from the likelihood ratio test. The negative trend was clearly observed when temperature

@ERSpublications

In this national ecological study across 154 Chinese cities, the ambient temperature was found to have a nonlinear negative association with COVID-19 transmissibility https://bit.ly/3esw8rc

Cite this article as: Ran J, Zhao S, Han L, et al. A re-analysis in exploring the association between temperature and COVID-19 transmissibility: an ecological study with 154 Chinese cities. Eur Respir J 2020; 56: 2001253 [https://doi.org/10.1183/13993003.01253-2020]. 

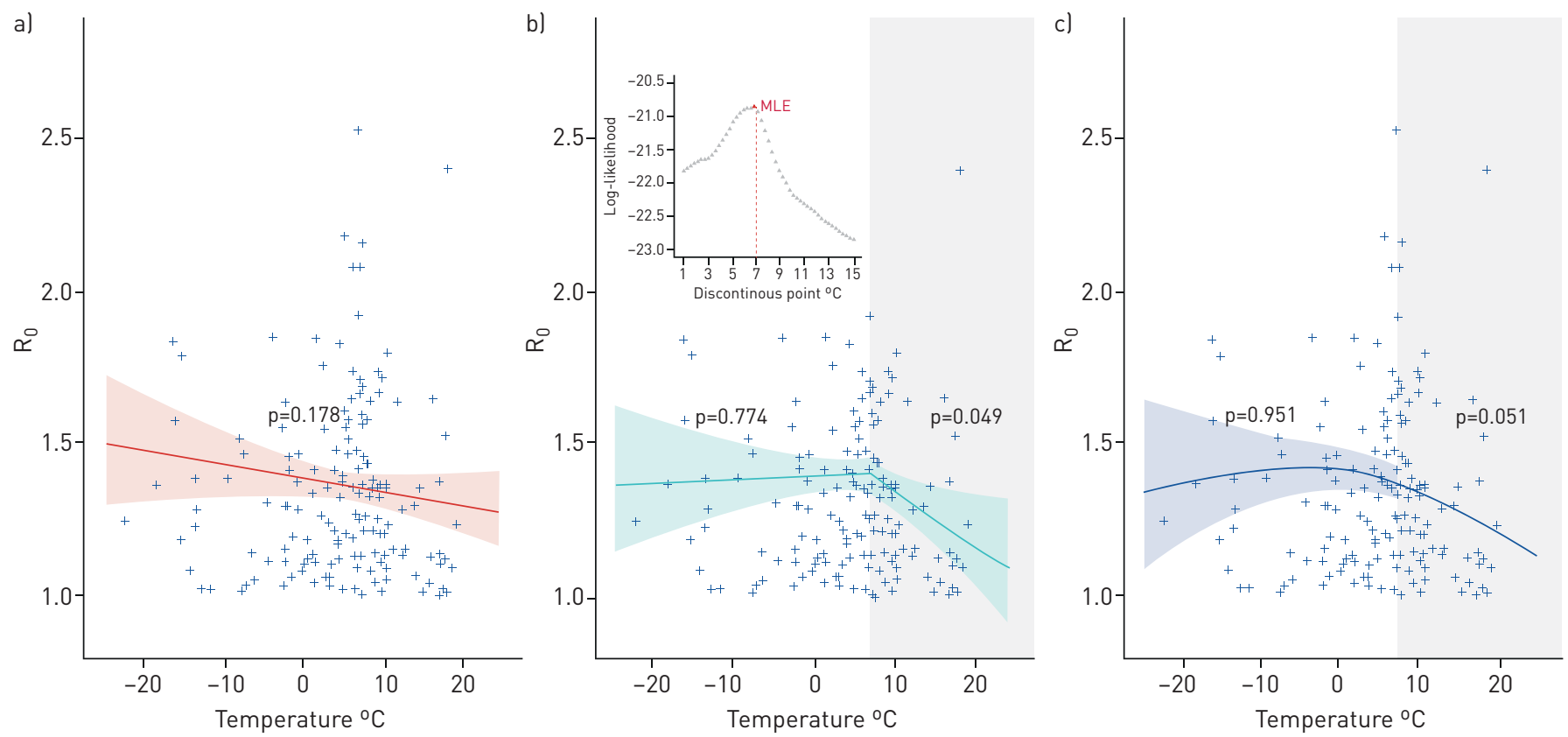

FIGURE 1 Association between temperature and coronavirus disease 2019 transmissibility $\left(R_{0}\right)$ across 154 Chinese cities. a) Multiple linear regression ( $p=0.178 ; A I C=53.82)$. b) Linear regression with a discontinuous point at $7^{\circ} \mathrm{C}\left(p=0.049\right.$ when temperature is over $\left.7^{\circ} \mathrm{C} ; A I C=51.78\right)$. The inserted panel shows the likelihood profile of the discontinuous point and its maximum likelihood estimate (MLE). The red triangle is the $\mathrm{MLE}$ at $7^{\circ} \mathrm{C}$, and the grey triangles are the input samples. c) Spline regression with a knot at $7^{\circ} \mathrm{C}$ ( $\left.\mathrm{AIC}=53.41\right)$. AIC: Akaike information criterion.

is higher than $7^{\circ} \mathrm{C}$ (figure 1c), which appears similar to the patterns of the discontinuity regression (figure 1b). We further tested the interaction between temperature and relative humidity on the COVID-19 transmissibility in the model. No clear interaction effect (or effect modification) was observed in terms of the p-values $(>0.050)$ from the likelihood ratio test. We further adjusted for fine particulate matter $\left(\mathrm{PM}_{2.5}\right)$ in the model, and we still observed the negative trend between temperature and $R_{0}$ values when temperature was over $7^{\circ} \mathrm{C}$.

Temperature could relate to viral transmissibility due to its impacts on virus viability and host immunity. Viruses are more stable at low temperature, and hosts may be more susceptible to the disease due to weakened immune protection [8]. Similar findings were also observed in extended experimental studies on other coronaviruses $[9,10]$. Due to the fact that major outbreaks in China ended before April 2020, the mean temperatures were below $15^{\circ} \mathrm{C}$ for most Chinese cities, i.e. $91 \%$ and $89 \%$ of the cities in our analysis and that by $\mathrm{YAO}$ et al. [1], respectively, and $80 \%$ and $81 \%$ were below $10^{\circ} \mathrm{C}$. Hence, we suspected a more evidently negative association might be observed from the COVID-19 epidemics in a warmer context.

Caution should be taken when interpreting the results. First, the temperature cut-off, i.e. $7^{\circ} \mathrm{C}$, was selected based on the modelling process. While uncovering its practical significance is limited in the current ecological study design, we emphasised the likelihood of the structural change in the association between ambient temperature and COVID-19 transmissibility. Further studies are required for exploring and quantifying the dose-response relationship between ambient temperature and COVID-19 transmissibility in more complex contexts. Second, since the outbreak will not occur with $R_{0}<1$ theoretically, only sporadic cases may be observed. In this situation, a precise estimation of $R_{0}$ would be difficult, and thus only cities with $R_{0}>1$ were included in our analysis. This stratification may limit our conclusion within the epidemic regions. Third, the potential impact of temperature is rather restricted since the change in temperature may solely contribute to $11.2 \%$ variability of $R_{0}$, in terms of the McFadden's pseudo-R-squared. Hence, we remark that control strategies for the COVID-19 pandemic barely relying on environmental factors are unlikely to succeed. Moreover, temperate and subtropical climates dominate most of China (the hardest-hit cites lie in similar latitude). The impact of temperature on the COVID-19 transmission in tropics remains uncertain due to the heterogeneity in climate. Last, the ecological study is not enough to evidence the causal relationship because of the residual confounding by other covariates, such as social distancing and age distribution.

We suggest that the existence of an association between COVID-19 transmissibility and ambient temperature should be examined and reaffirmed with systematic and comprehensive settings, and we argue that a nonlinear association might exist, similar to that seen for influenza. Studies with longer observation periods and stronger evidences are warranted to further test and verify this. 
Jinjun Ran ${ }^{1,7}$, Shi Zhao $\oplus^{2,3,7}$, Lefei Han ${ }^{4}$, Gengze Liao ${ }^{2}$, Kai Wang ${ }^{5}$, Maggie H. Wang ${ }^{2,3}$ and Daihai He $\oplus^{6}$

${ }^{1}$ School of Public Health, Li Ka Shing Faculty of Medicine, University of Hong Kong, Hong Kong Special Administrative Region, China. ${ }^{2} \mathrm{JC}$ School of Public Health and Primary Care, Chinese University of Hong Kong, Hong Kong Special Administrative Region, China. ${ }^{3}$ CUHK Shenzhen Research Institute, Shenzhen, China. ${ }^{4}$ School of Nursing, Hong Kong Polytechnic University, Hong Kong Special Administrative Region, China. ${ }^{5}$ Dept of Medical Engineering and Technology, Xinjiang Medical University, Urumqi, China. ${ }^{6}$ Dept of Applied Mathematics, Hong Kong Polytechnic University, Hong Kong Special Administrative Region, China. ${ }^{7}$ J. Ran and S. Zhao contributed equally, and thus they are considered as joint-first authors.

Correspondence: Daihai He, Dept of Applied Mathematics, Hong Kong Polytechnic University, Hong Kong, China. E-mail: daihai.he@polyu.edu.hk

Received: 18 April 2020 | Accepted after revision: 17 June 2020

Author contributions: J. Ran and S. Zhao conceived the study and carried out the analysis. L. Han collected the data. J. Ran and S. Zhao drafted the letter. J. Ran, S. Zhao and D. He discussed the results. All authors critically read and revised the letter and gave final approval for publication.

Conflict of interest: J. Ran has nothing to disclose. S. Zhao has nothing to disclose. L. Han has nothing to disclose. G. Liao has nothing to disclose. K. Wang has nothing to disclose. M.H. Wang is a shareholder of Beth Bioinformatics Co., Ltd. D. He reports grants from Alibaba (China) Co. Ltd collaborative research grant, outside the submitted work.

Support statement: D. He was supported by General Research Fund (Grant Number 15205119) of the Research Grants Council (RGC) of Hong Kong, China and an Alibaba (China) Co. Ltd Collaborative Research project. The funding agencies had no role in the design and conduct of the study; collection, management, analysis, and interpretation of the data; preparation, review, or approval of the manuscript; or decision to submit the manuscript for publication. Funding information for this article has been deposited with the Crossref Funder Registry.

\section{References}

1 Yao Y, Pan J, Liu Z, et al. No Association of COVID-19 transmission with temperature or UV radiation in Chinese cities. Eur Respir J 2020; 55: 2000517.

2 Lowen AC, Mubareka S, Steel J, et al. Influenza virus transmission is dependent on relative humidity and temperature. PLoS Pathog 2007; 3: 1470-1476.

3 Barreca AI, Shimshack JP. Absolute humidity, temperature, and influenza mortality: 30 years of county-level evidence from the united states. Am J Epidemiol 2012; 176: 114-122.

4 Ferretti L, Wymant C, Kendall M, et al. Quantifying SARS-CoV-2 transmission suggests epidemic control with digital contact tracing. Science 2020; 6936: eabb6936.

$5 \mathrm{Li} \mathrm{Q}$, Guan X, Wu P, et al. Early transmission dynamics in Wuhan, China, of novel coronavirus-infected pneumonia. N Engl J Med 2020; 382: 1199-1207.

6 Wang J, Tang K, Feng K, et al. High temperature and high humidity reduce the transmission of COVID-19. SSRN Electron J 2020; preprint [https://doi.org/10.2139/ssrn.3551767].

7 Wang M, Jiang A, Gong L, et al. Temperature significantly change COVID-19 transmission in 429 cities. medRxiv 2020; preprint [https://doi.org/10.1101/2020.02.22.20025791].

8 Lipsitch M, Viboud C. Influenza seasonality: lifting the fog. Proc Natl Acad Sci USA 2009; 106: 3645-3646.

9 Chan KH, Peiris JSM, Lam SY, et al. The effects of temperature and relative humidity on the viability of the SARS coronavirus. Adv Virol 2011; 2011: 734690.

10 Casanova LM, Jeon S, Rutala WA, et al. Effects of air temperature and relative humidity on coronavirus survival on surfaces. Appl Environ Microbiol 2010; 76: 2712-2717. 